\title{
Machine Learning Regression Guided Thermoelectric Materials Discovery - A Review
}

\author{
Guangshuai Han, ${ }^{1, \#}$ Yixuan Sun, ${ }^{2, \#}$ Yining Feng, ${ }^{1}$ Guang $\operatorname{Lin}^{2,3,{ }^{*}}$ and $\mathrm{Na} \mathrm{Lu}{ }^{1,3, *}$
}

\begin{abstract}
Thermoelectric materials have increasingly been given attention recently due to their potential of being a solid-state solution in converting heat energy to electricity. Good performing thermoelectric materials are expected to have high electrical conductivity and low thermal conductivity which are usually positively correlated. This poses a challenge in finding suitable candidates. Designing thermoelectric materials often requires evaluating material properties in an iterative manner, which is experimentally and computationally expensive. Machine learning has been regarded as a promising tool to facilitate material design thanks to its fast inference time. In this paper, we summarize recent progress and present the entire workflow in machine learning applications to thermoelectric material discovery, with an emphasis on machine learning regression models and their evaluation.
\end{abstract}

Keywords: Machine learning; Thermoelectric materials; Materials discovery; Regression.

Received date: 7 March 2021; Accepted date: 10 April 2021.

Article type: Review article.

\section{Introduction}

Due to the increasing demand for clean and renewable energy, discovering high-performance thermoelectric (TE) materials has received considerable critical attention..$^{[1,2]}$ The TE effect enabled the generation of versatile electric energy from ubiquitous heat energy. To maximize the TE effect, the TE materials are desired to have high Seebeck and high electrical conductivity but low thermal conductivity, which is quite challenging since these two properties are usually positively correlated. ${ }^{[3-5]}$ TE properties are complex transport properties, which are hard to be determined both from experimental measurement and theoretical calculation. ${ }^{[6-8]}$ To address the current bottleneck of the TE technology, uncover new TE materials with desired performance is urgently needed. Thanks to the everlasting contribution from the TE community, tremendous in-depth research with fruitful research data makes the data-driven approach achievable.. ${ }^{[9,10]}$ The

\footnotetext{
${ }^{1}$ Lyles School of Civil Engineering, Sustainable Materials and Renewable Technology (SMART) Lab, Purdue University, West Lafayette, IN 47906, USA.

2 School of Mechanical Engineering, Purdue University, West Lafayette, IN 47906, USA.

3 Center for intelligent infrastructure, Purdue University, West Lafayette, IN 47906, USA.

\#These authors contributed to this work equally.

*E-mail: guanglin@purdue.edu (G. Lin), luna@purdue.edu (N. Lu)
}

emergence of artificial intelligence (AI) methods shed new light on the further usage of these resources. The combination of big data and machine learning algorithms could maximally explore the potential of the existing research data and enhance the discovery of the new TE materials. ${ }^{[1]}$ These data-driven methods enable high TE performance materials discovery with high efficiency and low experimental cost.

The data-driven methods and machine learning have been widely employed in materials science for predicting the mechanical properties and responses, material screening, and facilitating design. ${ }^{[12,13]}$ Nonetheless, the number of studies of machine learning methods specifically focused on TE materials is limited. In a survey of machine learning approaches for TE material research, ${ }^{[14]}$ the authors summarized machine learning techniques used in the existing studies, which included several both supervised and unsupervised learning models and a Bayesian optimization framework. Also, available databases that could be used for developing machine learning models and model input feature representation were discussed in the work. However, the means to generate usable data and evaluating and comparing trained models are missing. In this work, we present the entire workflow to TE material discovery using machine learning with a focus on regression models. Furthermore, we discuss the evaluation metrics for regression models and present readers with suitable scenarios for the metrics. To the best of our knowledge, this is the first comprehensive survey 


\section{-}
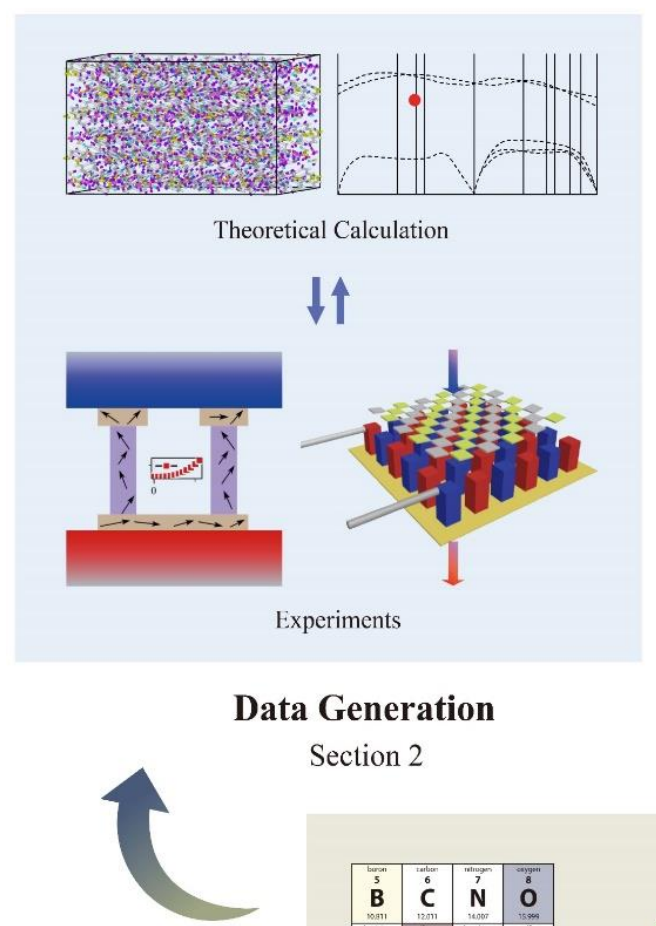

Data Generation

Section 2

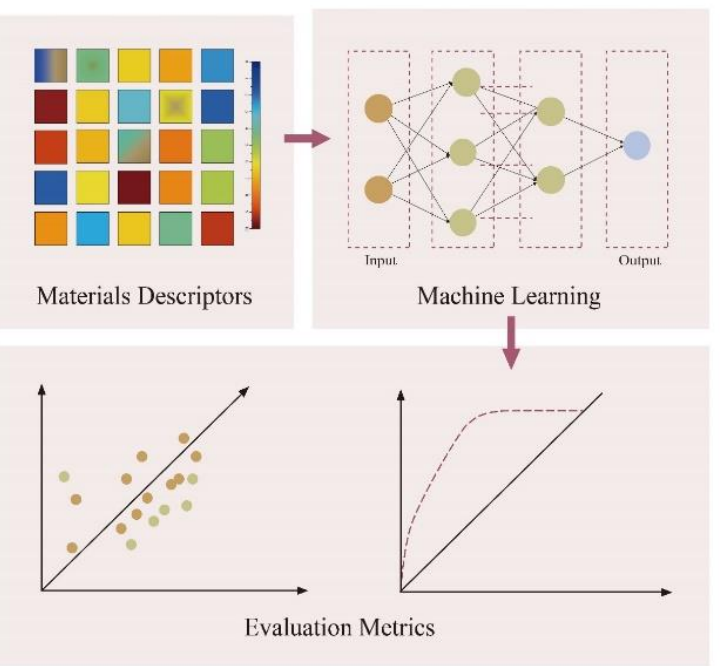

Model Training

Section 3 to 5

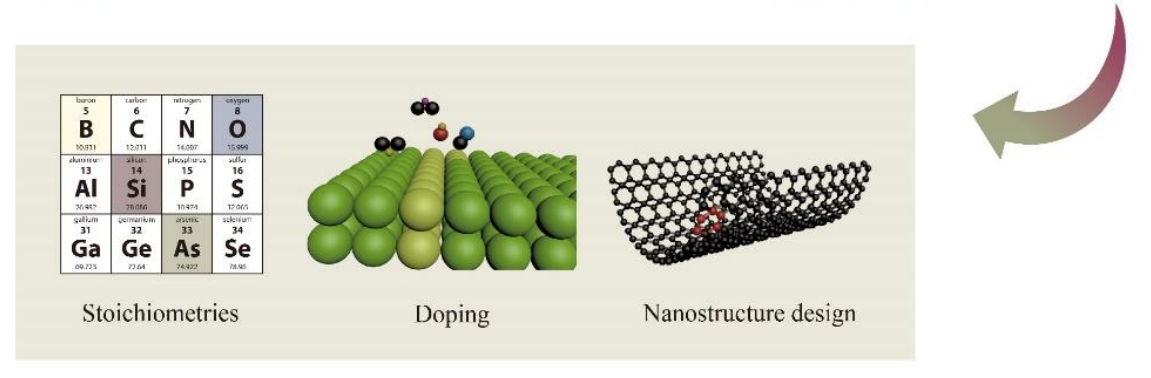

Material Discovery

Section 6

Fig. 1 The logistic structure of machine learning guided TE materials discovery. This machine learning guided TE materials discovery is described as a lopped investigation process. Start from the blue area, the TE database is generated by a continuous contribution from the TE community employing experiments and theoretical calculation. The data generation is described in section 2 . The red area denotes the model training which is how to develop the machine learning based TE materials discovery tools by learning from the TE database. The input descriptors, machine learning technique, and model evaluation are discussed separately in sections 3 to 5 . The yellow area expresses the guidance from the TE materials discovery tools. The newly discovered materials can be further utilized as new data to improve the performance of the TE materials discovery tools, which build a cyclic structure.

to machine learning regression aided TE material discovery, which includes data generation, material descriptor selection, machine learning regression models, and model evaluation.

Fig. 1 shows the logistic structure of this review paper. The machine learning for TE materials discovery is presented as a looped procedure. Start from section 2, we firstly go through the TE data generation from the existing research. Later, the model training is elaborated by three sections. The commonly used descriptors with their importance are illustrated in section 3. The descriptors selection and treatment are firstly summarized. Different types of machine learning techniques in TE materials with the machine learning model evaluation methods are narrated respectively in section 4 and section 5 .
Lastly, the functionality of the machine learning based TE materials discovery tool is summarized in the last section. The valuable data from the materials discovery tool could be cyclically contributing to the data generation as the new resources. Further work is also suggested at the end of the review paper.

\section{Data Generation}

\subsection{Experimental characterization}

Machine learning requires preexisting knowledge (database) for models to learn from. For the machine learning guided TE materials discovery, the materials database should be labeled with the desired TE properties. The TE property is dominated 
by three combinatorial materials properties, which are Seebeck coefficient, electrical conductivity, and thermal conductivity. The quality of TE materials is normally evaluated by a dimensionless figure of merit $z T$, which is defined as following ${ }^{[1]}$

$$
z T=\frac{S^{2} \sigma}{k_{T}} T
$$

where $S$ is the Seebeck coefficient, $\sigma$ is the electrical conductivity, $k_{T}$ is the total thermal conductivity, $T$ is the temperature. The thermal conductivity $k_{T}$ is the sum of the electronic contribution $k_{e}$ and lattice contribution $k_{l}$. Since these three combinatorial parameters are affected by each other, the biggest challenge for the development of TE materials is to optimize these three parameters. Accordingly, these three combinatorial parameters are the most popular labels for the TE machine learning model. As the phonon and electron transport controls the TE materials performance, the band structure, bandgap, and phonon dispersion are considered as preferable labels as well. ${ }^{[15,16]}$

The development of a machine-learning-based material discovery tool relies on a large volume of data, from which the learning process can result in reliable relations in the input and output pairs. The most common way for obtaining these TErelevant data for different materials is experimental characterization. The collection of the materials' TE-related experimental results from different research groups are valuable resources for developing machine learning based TE materials discovery. The UCSB database is one of the most comprehensive TE materials databases. ${ }^{[17]}$ The UCSB database contains the TE relevant properties at several temperatures for over 1000 different compounds by abstracting information from over 100 publications. By combining with appropriate visualization tools as shown in Fig. 2(a), the UCSB database can provide researchers an efficient approach to develop new TE materials. From the material design point of view, this database can offer constructive suggestions, such as all high$z T$ materials are found in the metallic conduction region 0.001 $\Omega \mathrm{cm}$ to $0.01 \Omega \mathrm{cm}$. The potential of the UCSB database was further exploited by the machine learning technique. Furmanchuk et al.$^{[18]}$ developed a machine learning model to predict the Seebeck coefficient at four different temperatures of synthesized materials by utilizing the UCSB database. A high prediction accuracy with the r-square round from 0.74 to 0.84 was obtained when predicting the Seebeck coefficient of materials with different chemical compositions.

Doping is a well-known strategy to improve the materials TE properties. ${ }^{[19-22]}$ Wang et al. ${ }^{[23]}$ adopted a machine learning technique to optimize the $\mathrm{Cu}$ content in $\mathrm{Cu}$-doped $\mathrm{Bi}_{2} \mathrm{Te}_{2.85} \mathrm{Se}_{0.15}$. The experimentally measured $\mathrm{zT}$ with varying $\mathrm{Cu}$ content is used as the label for the machine learning artificial neural network (ANN). The obtained model with a correlation coefficient of 0.99 indicates excellent accuracy. However, over-fitting was observed during the zT prediction due to the limited sample size. It is concluded that a sufficient amount of data is required for the present research.
Hou et al. adopt the machine-learning-based framework to find the optimal $\mathrm{Al} / \mathrm{Si}$ ratio in $\mathrm{Al}_{2} \mathrm{Fe}_{3} \mathrm{Si}_{3}$ for TE applications by using the workflow presented in Fig. 2(c). ${ }^{[24]}$ The initial machine learning model was obtained by serval $\mathrm{Al}_{23.5+\mathrm{x}} \mathrm{Fe}_{36.5} \mathrm{Si}_{40-\mathrm{x}}$ composition. The experimentally measured power factor for the un-synthesized materials predicted by the machine learning model served as additional data to improve the machine learning model. The optimal $\mathrm{Al} / \mathrm{Si}$ ratio was obtained after the repeat iteration (as shown in Fig. 2(c)). The materials with optimal ratio increase the power factor by about $40 \%$. Machine learning techniques have also been used to identify the optimal the optimal internal strain for TE materials. Saaki et al., used the machine learning technique (as shown in Fig. 2(d)) to find the relation between XRD (Xray diffraction) and the materials' Seebeck coefficient. The trained model suggests that the optimal strains of 3-4 \% and 1$2 \%$ along the a- and c-axis, can significantly improve the Seebeck coefficient. ${ }^{[25]}$ In summary, the TE properties from experimental characterization are an important data source for the machine learning process. The developed machine learning model is a powerful tool for the stoichiometry and nanostructure optimization for TE materials.

\subsection{Theoretical calculation}

In addition to the TE data from experimental characterization, atomic-scale materials first principle calculation is another way to attain materials' TE properties. Compare to the database from the experimental characterization, the computational results have the merit of standardization, which means the TE results would not suffer from the influence of the equipment, human, and measuring error. Based on the Boltzmann transport equation, the electronic thermal conductivity, lattice thermal conductivity, electrical conductivity, and Seebeck coefficient can be obtained from the computational results. ${ }^{[26,27]}$

Even though the first principle calculation equation can provide accurate and reliable TE data, this approach is dramatic computationally expensive. ${ }^{[28]}$ Therefore, it is hard to use the first principal calculation to satisfy the large-dataamount-requirement for machine learning. This dilemma has been addressed by the development of the high-throughput first principle calculation. ${ }^{[29]}$ On high-throughput first principle, the computational cost can be dramatically reduced with slightly sacrificed accuracy. ${ }^{[30-32]}$ The results from the high-throughput first principle calculation can be saved in large materials databases for further utilization, such as fast materials screening, machine learning. ${ }^{[33]}$ By adopting highthroughput first principle calculation, Ricci et al. present the largest computational database of transport properties of around 48000 materials. ${ }^{[34]}$ Fig. 2(b) shows the overview of the dataset. The TE-relevant properties, such as electronic conductivity, electronic thermal conductivity, and Seebeck coefficient are calculated from materials' band structure by using Boltzmann transport theory. The data is saved on the Materials Project website. The database also covered the 


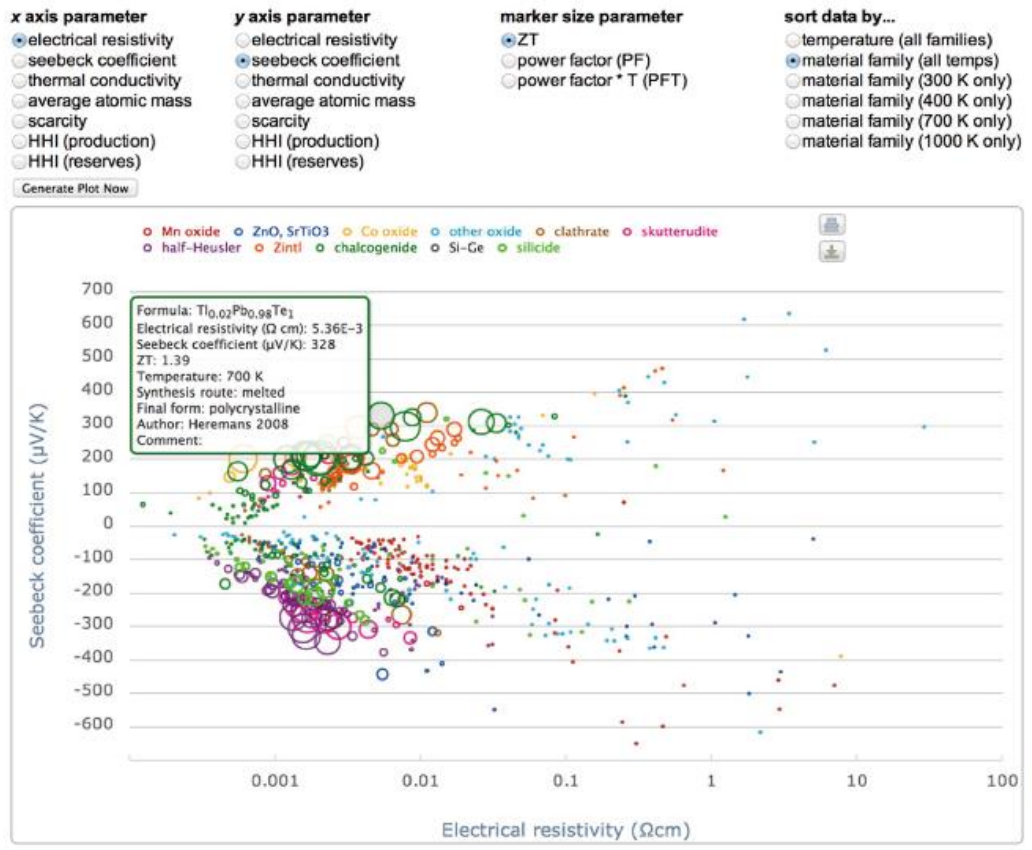

Material families: Mn oxides, $\mathrm{Co}$ oxides, $\mathrm{ZnO}$ and $\mathrm{SrTiO}_{3}$, other oxides, chalcogenides, clathrates, skutterudites, half-Heuslers, Zintis, $\mathrm{Si}$ and $\mathrm{Ge}$, Sllicide

Supported by the National Science Foundation by NSF DMR 1121053 (MRSEC)

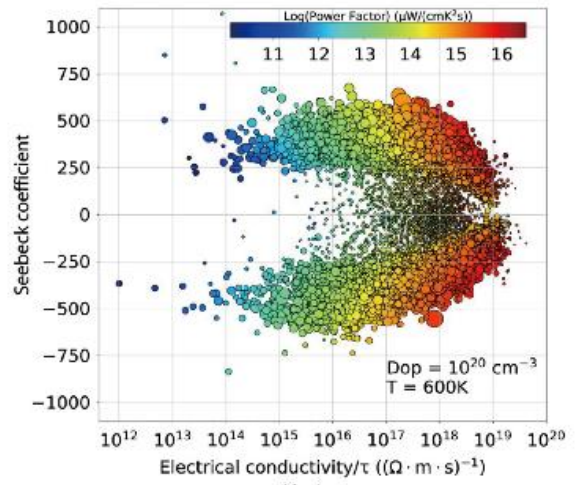

(b)

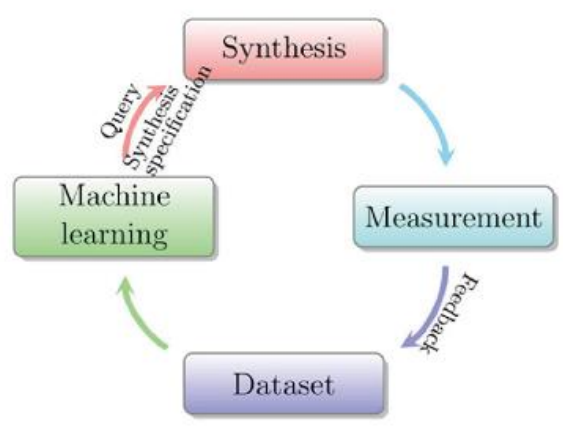

(c)

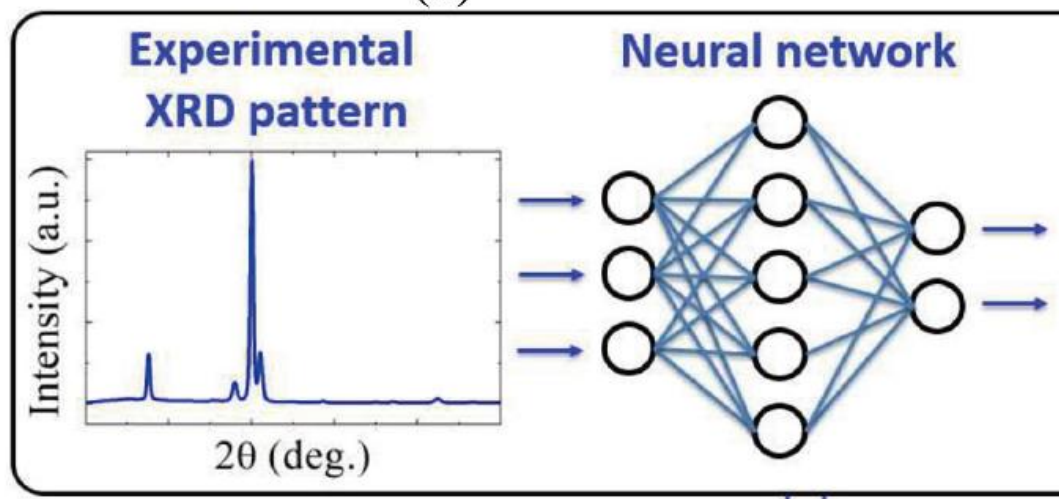

Experimental Seebeck coefficient

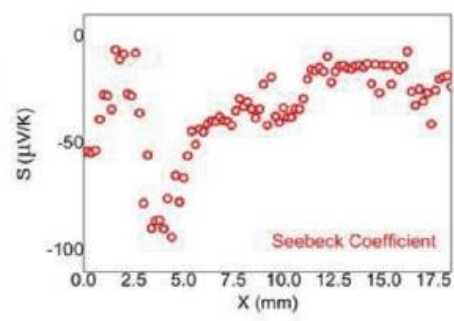

strain engineering

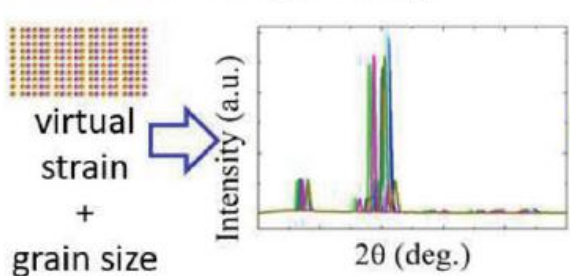

Structure $\rightarrow$ XRD

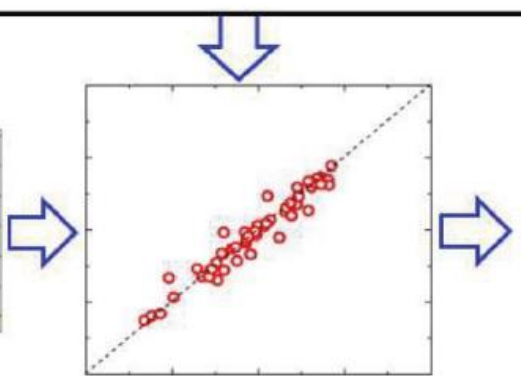

Prediction model

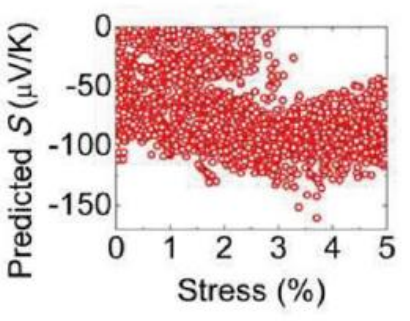

High Seebeck structure

(d)

Fig. 2 (a) Screenshot of the web-based visualization tool for the UCSB dataset. (Reprinted with permission from Ref.[17], Copyright 2013, American Chemical Society) (b) the TE dataset provided by Ref [34]. The color represents the power factor and the point size is used for band gap. (c) Framework for the design of $A l_{23.5+x} F_{36.5} S i_{40-x}$ toward an optimized power factor (PF). (Reprinted with permission from Ref [24]) (d) Diagram showing the use of XRD data to design TE materials via machine learning. The prediction model is first trained using the experimental XRD patterns and measured Seebeck coefficient. The trained model is then used to screen the XRD candidates containing random combinations of internal stress and grain size. The optimal structures with the best Seebeck coefficient are finally selected. (Reprinted with permission from Ref.[25], Copyright 2020, American Chemical Society). 
materials' transport properties at various fixed doping carrier concentrations, Fermi level energies, and temperatures. These computation results have been proved to have a fair agreement with the experimentally measured maximum Seebeck coefficient by further investigation. ${ }^{[35]}$ This trustworthy and abundant database is a valuable resource for machine learning based TE materials discovery techniques. The publicly available JARVIS-DFT database contains TE performance data of 900 two-dimensional materials and 36000 threedimensional from high-throughput density functional theory (DFT) calculations. ${ }^{\left[{ }^{[3]}\right.}$ In addition to the electronic thermal conductivity, electrical conductivity, Seebeck coefficient, the JARVIS-DFT further the lattice thermal conductivity. This data was further used to develop the machine learning classification model to pre-screen materials with good TE properties. The accuracy of the classification model was evaluated by the area under the curve (AUC) of the receiver operating characteristics curves. The lowest AUC of 0.8 and the highest AUC of 0.96 were reported for predicting materials Seebeck coefficient and Power factor 0.001, which denotes an adequate accuracy for pre-screen TE materials.

\section{Descriptors}

\subsection{Critical role of material descriptors}

By means of experimental characterization and theoretical calculation, numerous materials have been discovered and studied. Accordingly, the obtained data, which has been proved as a valuable resource for machine learning of materials properties, increase rapidly with time. Finding meaningful parameters to represent materials' unique physical and chemical properties, is one of the most essential steps for machine learning of materials properties. These parameters are termed descriptors and constitute the input for the machine learning process. A straightforward example of materialrelated descriptors is the table row and column of the periodic table. ${ }^{[37]}$ The elements' uniqueness, such as the atomic number, number of electrons, and numbers of valence electrons, can be well categorized by the table row and column. Moreover, tracing back to the period when some of the elements have not been discovered, their physical and chemical properties can be magically predicted by this two-dimensional descriptor. However, it is more complicated to find suitable descriptors for materials with diverse atomic structures. Finding suitable descriptor generation methods for different materials has been continually studied with growing interest. ${ }^{[38-40]}$ Herein, we summarized the most recognized requirements of good descriptors for materials:

- Unique: the descriptors should uniquely characterize the materials which are representing.

- Continuous: the amount of materials' property differences need to be reflected by the descriptors in the same level.

- Computationally reasonable: the determination of the descriptors should require less computational or experimental cost than the determination of the desired materials' properties, and preferably, no additional computational or experimental effort is needed.

- Effective: the descriptors should comprehensively represent the physical and chemical characteristics with the lowest dimensionality.

\subsection{Descriptors for TE importance}

The study of TE properties is a multidisciplinary topic..$^{[41]}$ The performance of TE materials is always controlled by the lattice vibrations, carrier transport, electronic orbitals, spin properties, etc. ${ }^{[41]}$ Based on this, Wang et al. listed the relevant descriptors for TE properties. ${ }^{[42]}$ The density-of-states effective mass, charge-carrier mobility, and Pauling electronegativity are commonly used descriptors for TE materials. Many other materials properties, such as lattice thermal conductivity, electron-phonon interaction, have also been proved to have an extensive effect on thermoelectricity. The complex interrelation of TE-related materials properties signifies that it is hard but unavoidable to properly select the descriptors for machine learning based TE materials discovery.

Although the above-mentioned descriptors are strongly related to the TE properties, such as electrical conductivity, thermal conductivity, and Seebeck coefficient, some of the descriptors cannot be directly used as input for machine learning. Most of the listed descriptors are not originally available and the determination of these descriptors is experimentally and computationally expensive. These descriptors should be further represented by more elementary descriptors, which are categorized into chemical descriptors and structural descriptors. Stoichiometry optimization is always the goal of using machine learning to discover good TE materials. The chemical descriptors contain the chemical composition information of the material and are further enriched by the atomic and macro properties of the elements within the material. Since this information of elements has been well studied and available in a tremendous publicly available database, these elementary descriptors perfectly meet the above-mentioned requirements for good descriptors. Bassman et al. utilized the atomic properties: electronegativity, first ionization potential, and atomic radius as the descriptors to build the machine learning regression model to predict the layered materials' bandgap and TE properties. ${ }^{[43]}$ The regression model displays a high accuracy for predicting the materials' TE properties. The element's position in the periodic table has also been used to build the machine-learning engine for TE materials discovery. ${ }^{[4]}$ In addition to the location of elements in the periodic table and atomic properties of elements, Furmanchuk et. al further included the experimentally measured macro properties of pure elements, such as thermal conductivity, electric conductivity, and density, to build the machine learning model to predict the materials' Seebeck coefficient at a different temperature. ${ }^{[40]}$ The obtained regression has a correlation coefficient as high as 0.91 . This study further analyzed the top descriptors contributing to the Seebeck coefficient prediction. The experimentally measured 
pure elements' thermal conductivity shows the top contributions since the thermal conductivity of pure elements provides indirect information on the electronic transport properties of the compound. As for the atomic properties of elements, the electron affinity, atomic number, and electronegativity make a great contribution to the Seebeck coefficient prediction.

The structural descriptors represent how the elements within the materials are arranged in the space. Although the chemical descriptors have been successfully applied for various tasks of machine learning materials discovery, the structural information also strongly affects the thermoelectricity of materials and shouldn't be ignored. Choudhary et al. developed classical force-field-inspired descriptors (CFID) which combined the chemical descriptors and structural descriptors for machine learning usage. ${ }^{[45]}$ The structural descriptors, such as radial distribution function, nearest-neighbor distribution, and angle and dihedral distribution show almost equal importance for accurately the materials Seebeck coefficient and power factor. ${ }^{[36]}$ The total importance of the chemical descriptors is lower than which of the structural descriptors. This results also in contrast with the importance distribution for the machine learning formation energy prediction model, where the chemical descriptors display better importance than the structural descriptors. Thus, both chemical descriptors and structural descriptors should be utilized when developing the machine learning model for TE materials discovery.

\subsection{Descriptor treatment}

The entire descriptors for each material used within a machine learning model training task should be transferred into a feature vector/matrix with the same dimension. ${ }^{[21]}$ Normally, the descriptors should be organized based on a standard way. For instance, the feature vector adopted by Bassman et al. order the descriptors based on the layered structure of the materials with $A B_{2}$ format (e.g. $\mathrm{MoSe}_{2}, \mathrm{WTe}_{2}, \mathrm{MoS}_{2}$ ). The number of atoms within one single molecular is the same. ${ }^{[43]}$ Thus, the dimension of the organized feature is the same. A similar approach has been applied for the materials with the same $\mathrm{AB}_{2} \mathrm{C}$ format to generate the feature vector. ${ }^{[4]}$ However, for most circumstances, dimension control is still a challenge since different materials contain different amounts and types of atoms within one molecular. Several methods have been proposed and applied to feature engineering for the machine learning of TE materials. The statistical approach is one of the effective ways to address this challenge. Using the statistical model, such as sum, maximum, and mean absolute deviation from mean will result in the ultimately organized feature vector have the same dimension. ${ }^{[21]}$ The statistics-based feature vector shows excellent performance when predicting the Seebeck coefficient of materials. The structural descriptors are normally in the format of a matrix, such as the Coulomb matrix, Ewald sum matrix, etc. ${ }^{[39]}$ For the matrix descriptors, a common way to achieve a standard size of geometries with different amounts of atoms is by introducing zero-padding. The maximum size of matrices will be pre-determined based on the materials involved in one machine learning model training task. ${ }^{[40]}$ Then, the extra space will be set to zero. With these approaches, the materials information can be transformed into feature vectors/matrices which are suitable for machine learning usage.

\section{Machine Learning Applications in Thermoelectric Materials}

The rapid development of data-driven/machine learning methods has enabled learning complex functions from data in material science. Different from the first principle-based methods, data-driven methods do not require physical models or complex system solvers, and thus have the potential of performing fast prediction and learning unknown physics from the data. In the field of TE materials, most machine learning applications are on selecting or engineering proper descriptors, predicting the property of interest from various descriptors without imposing physical models, and facilitating discovering material with desired properties. In this section, we organize the applications of machine learning methods for TE materials into feature learning and regression. Among the machine learning regression models, we further categorize the current work into classic statistical learning and deep learning, and then point out the advantages and disadvantages of those methods and summarize the future work.

\subsection{Feature learning}

To sufficiently and accurately predict material properties, it is important to choose the right descriptors containing satisfactory information for the corresponding properties. While there is a tremendous number of descriptors to choose to account for the materials' nature, it is not obvious how to select the right ones. Besides, machine learning methods, in general, require a much greater number of instances than the number of attributes/descriptors to prevent overfitting, so that they can generalize well on the new unseen data. To address this problem, work has been done on either selecting the informative descriptors out of the candidate pool or transforming the descriptors to another set of features with lower dimensions, meanwhile, preserving the original information. These two approaches are feature selection and feature engineering, respectively. Data-driven feature selection and engineering do not require domain knowledge with which one can take out or combine descriptors based on their expertise. Instead, feature selection and engineering can be performed in a supervised or unsupervised manner. The current common methods on data-driven feature selection and engineering for TE materials are Pearson correlation, principal component analysis, and auto-encoders. They are described in more detail as follows.

\subsubsection{Pearson correlation}

Pearson's correlation or Pearson's correlation coefficient 


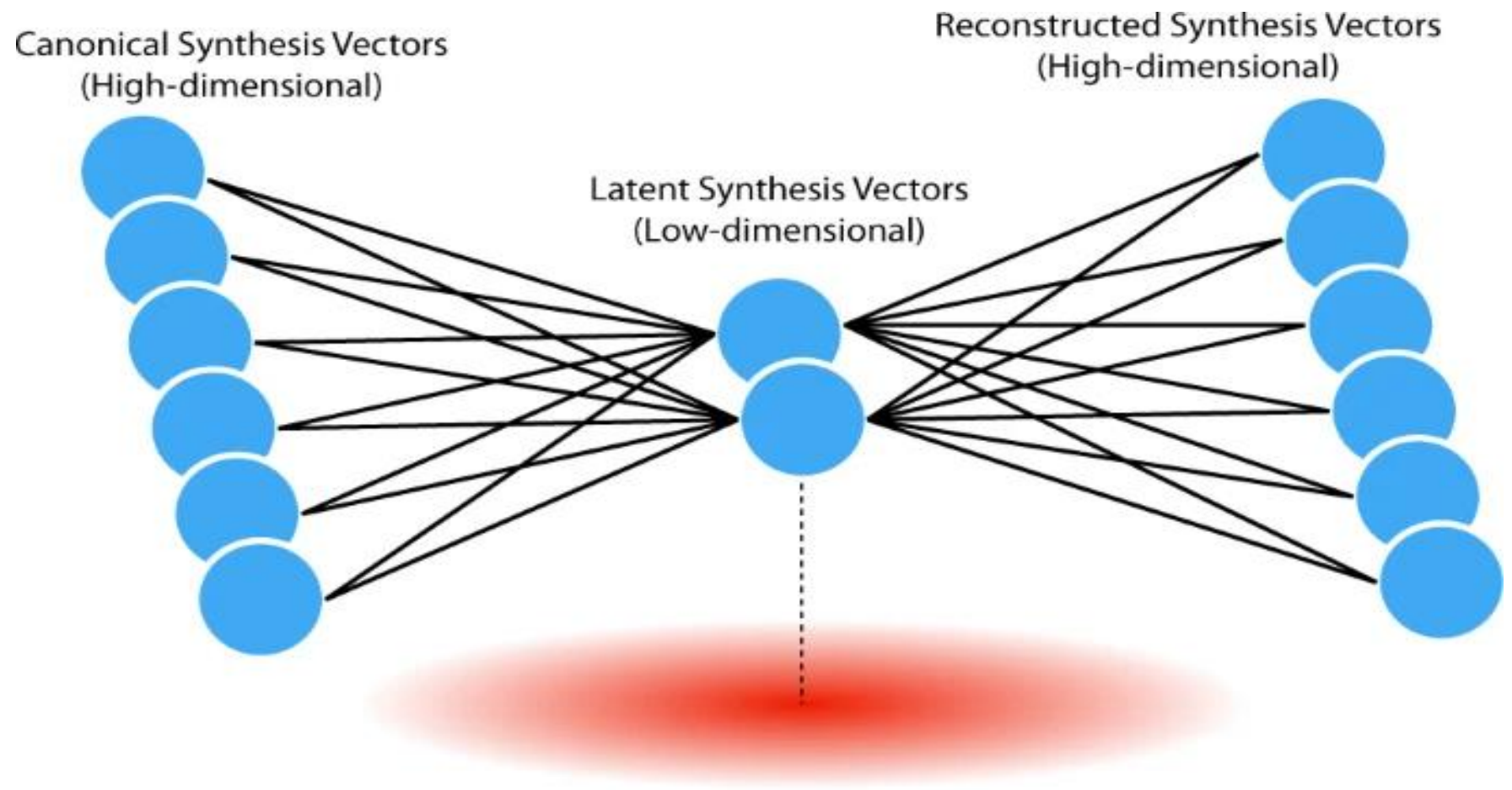

Low-dimensional Latent Synthesis Space

Fig. 3 The schematic of the variation auto-encoder. ${ }^{[50]}$ It learns a low-dimensional representation in the latent space from the original canonical synthesis vectors.

accounts for the linear relation between two random variables. It is defined as follows.

$$
\rho_{X, Y}=\frac{\operatorname{cov}(X, Y)}{\sigma_{X} \sigma_{Y}}
$$

where the $\operatorname{cov}(\cdot)$ represents the covariance of random variables $X$ and $Y$, and $\sigma_{X}, \sigma_{Y}$ are the standard deviation of the random variables. In the field of machine learning, Pearson's correlation is widely used for feature selection, where a positive near 1 value indicates that the two variables are highly linearly correlated and thus one can be taken out when fitting a machine learning model to prevent overfitting and computational cost. In addition, Pearson's correlation coefficient can be obtained between the material descriptors and desired properties, showing the predictive power of the descriptors in linearly predicting the target. In a study using linear regression to predict the material power factor, ${ }^{[46]}$ a positive Pearson's correlation coefficient was obtained between the power factor and both electronic band gap and carrier effective mass.

\subsubsection{Principal component analysis}

Principal component analysis (PCA) is a common linear dimension reduction technique used in transforming input features to a new coordinate so that one can reduce the number of predictors while preserving most of the original information. PCA takes linear combinations of the original features to form principal components (PCs), which capture the most possible variance. The first $\mathrm{PC}$ is the linear combination of the original features, containing the most variability out of all the possible combinations, and the second $\mathrm{PC}$ suggests the most variability among the rest combinations and so on. Therefore, the PCs of a dataset can provide the best linear approximations to it. The steps of conducting PCA are listed below.

- Standardize data.

- Create covariance matrix for eigen-decomposition.

- Calculate eigenvalues of eigenvectors of the covariance matrix to identify principal components.

- Select the optimal number of principal components.

More details of performing PCA can be found in a tutorial. ${ }^{[4]}$ PCA has been applied in TE material machine learning studies to reduce model input dimensions. In the study of predicting the force constants, Roekeghem et al. utilized PCA to transform the original descriptors and selected the first 10 PCs as the regression model input. ${ }^{[48]}$ Wagner et al. adopted PCA to transform highly correlated mode features and used the first 3 PCs to predict high-temperature Perovskites with decision trees. ${ }^{[49]}$

\subsubsection{Auto-encoders}

As an unsupervised learning method, serving the same purpose as PCA, auto-encoders are widely used to transform the original feature vectors to a lower-dimensional one, called the latent vector. Auto-encoders extract high-level representations of the original features via training a neural network where the dimensions of the input and output layers are the same. The objective of training an auto-encoder is to minimize the error of network output to its input. Fig. 3 shows the schematic of a variational auto-encoder used in 
compressing the material sparse synthesis representations into lower-dimensional space. Auto-encoders have the advantage of being able to transform the original feature vectors in a nonlinear manner. Kim et al. used a variation auto-encoder (VAE) to map 30 canonical material features to 10 latent variables in the latent space, ${ }^{[50]}$ and then utilized the trained VAE to generate potential synthesis parameters sets for virtual material screening.

\subsection{Regression}

The majority of the work on using machine learning models in the TE material field is regression. Regression models are the ones that output continuous real values given the input. The regression models represent the relationship between a single or multiple independent variable that can be quantitative or categorical and dependent variables that are quantitative. In the field of TE materials, researchers have been adopting welldeveloped regression frameworks to predict desired material properties, such as Seeback coefficient, figure of merit, thermal conductivity, etc, using well-curated and selected material descriptors. The regression models can be generally put into two categories: classic statistical learning models which are usually based on classic statistical learning theories, and deep learning models which are mainly based on neural network theories. This section is organized into two parts: the classic statistical learning and deep learning models.

\subsubsection{Classic statistical learning methods}

The classic statistical learning models, sometimes referred to as machine learning models (as opposed to deep learning) in general, are models that take a certain formulation based on statistical learning theories, where the model forms have been pre-determined. In a typical regression setting, the models tend to learn the underlying relations from outcome measurements, which can be quantitative or categorical. Compared to deep learning models which will be discussed in the second part of this section, the statistical learning models are easy to interpret due to the hypotheses the models are based on. Among the current studies of using statistical learning methods to predict TE material properties, linear regression, tree-based models, Gaussian processes, and support vector regression are most commonly used. In this section, we describe each of the algorithms in detail and discuss the advantages and disadvantages of those techniques.

\subsubsection{Linear regression}

A linear regression model assumes the regression function is linear in terms of the input variables $X_{1}, \ldots, X_{p}$, which can be the input material descriptors. The basic linear regression model has the form of:

$$
f(x)=\beta_{0}+\sum_{j=1}^{p} X_{j} \beta_{j}
$$

where $\beta_{0}$ is the learned bias term and $\beta_{j}$ is the learned weight corresponding to $X_{j}$. The linear models assume a linear relationship between the input independent variables and the output dependent variable, or the linear relationship is a reasonable approximation. Under this framework, the input variables can be the original quantitative or categorical values, such as the material descriptors, as well as the transformation of the original values such as log, square-root, polynomials, or other transformations. The parameters in Equation (3) are estimated by ordinary least square (OLS) which is minimizing the squared error, shown below.

$$
\min _{\beta} \sum_{i=1}^{N}\left(y_{i}-f\left(\mathbf{x}_{\mathbf{i}}\right)\right)^{2}
$$

where $N$ is the number of instances in the training data, $x_{i}$ is the feature vector of the $i$ th instance, and $y_{i}$ is the true value corresponding to the $i$ th instance. Minimizing Equation (4) to estimate the parameters is equivalent to solving a normal equation, shown in Equation (5).

$$
\widehat{\beta}=\left(\mathrm{X}^{\mathrm{T}} \mathrm{X}^{-1}\right) \mathrm{X}^{\mathrm{T}} \mathrm{y}
$$

where $X$ is the feature matrix and $Y$ is the true target vector. Although the basic form of linear regression is simple and useful in many scenarios, it can lead to overfitting when the learned parameters to some variables are unrealistically large fitting the training data but failing to match the unseen data. To address the high variability of the model, shrinkage methods can be used. The most commonly used regularization techniques are ridge regression and Lasso regression. Both ridge (Equation (6)) and Lasso regression (Equation (7)) add penalty terms, of which the amount is controlled by $\lambda$, to the OLS, limiting the magnitude of parameters to mitigate overfitting. While in ridge regression the model encourages small parameter magnitude using L2 norm, in Lasso regression the model encourages 0 values using L1 norm. Estimating the parameters in all three models can be done by solving the linear systems when the feature matrix is invertible. An alternative is to adopt gradient-based optimization techniques such as stochastic gradient descent to minimize Equation (4), (6), and (7) regardless of the feature matrix's invertibility.

$$
\begin{gathered}
\min _{\beta} \sum_{i=1}^{N}\left(y_{i}-f\left(\mathbf{x}_{\mathbf{i}}\right)\right)^{2}+\lambda \sum_{i=1}^{N} \beta_{j}^{2} \\
\min _{\beta} \sum_{i=1}^{N}\left(y_{i}-f\left(\mathbf{x}_{\mathbf{i}}\right)\right)^{2}+\lambda \sum_{i=1}^{N} \beta_{j}
\end{gathered}
$$

In the forms of applying linear regression models to $\mathrm{TE}$ materials, one can treat the material descriptors and their transformations as the input independent variables and the desired material properties as the output dependent variable. Wang et al. conducted linear regression analysis on predicting power factors of sintered powders and discovered that power factor is positively correlated with the electronic band gap and carrier effective mass. ${ }^{[46]}$ van Reokeghem et al. embedded the linear regression model in a material discovery scheme where they utilized PCA-transformed features to predict force constants of semiconducting oxides and fluorides with cubic perovskite structures at various temperatures. ${ }^{[48]}$ de Jong et al. constructed polynomial feature basis from composition and structural descriptors, and conducted Lasso regression with gradient boosting to predict elastic bulk and shear moduli of 


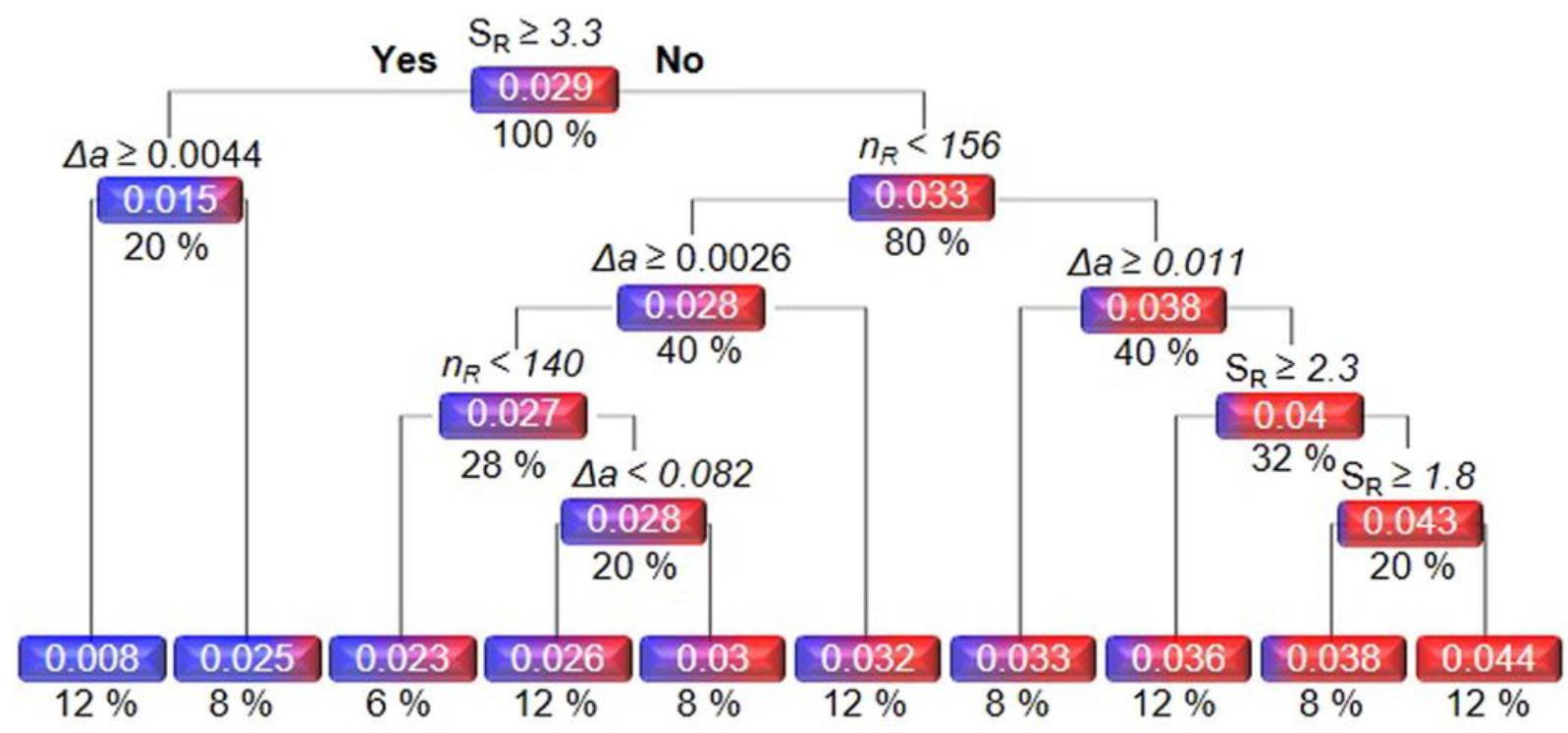

Fig. 4 Visualization of the decision tree regression model from[54] predicting thermopower of spin-driven TE effect materials. The model splits the data based on certain features, namely spin angular momenta $S_{R}$, orbital angular momenta $L_{R}$, lattice mismatch $\Delta \mathrm{a}$, and atomic weight $n_{R}$. Features with more importance in predicting thermopower appear at upper levels close to the root. The leaf nodes output the mean values in the training data of such splits.

polycrystalline compounds. ${ }^{[51]}$ They further used the welltrained model in screening superhard materials. Miller et al. explored the application of the linear regression model among other methods, for predicting carrier concentration range of diamond-like semiconductors. ${ }^{[52]} \mathrm{Li}$ et al. used Kernel Ridge Regression to predict the decomposition energy given the composition ionic radii and validated the trained model on the experimental perovskites formability. ${ }^{[53]}$ The success of the regression model demonstrated the machine learning methods applied on DTF-calculated data can guide the experimental engineering of stable perovskites. Iwasaki et al. ${ }^{[54]}$ used elastic net and quadratic polynomial lasso regression to predict the thermal power (Fig. 4).

Linear regression models are simple and easy to interpret, thus researchers should consider using them when first applying machine learning techniques to a specific regression problem. However, the assumption of a linear relation between the input features and output target only holds in limited scenarios. For TE materials where the input descriptors and material properties have complex non-linear relations, the models that can capture the non-linearity should be investigated. In the following sections, we discuss tree-based methods, Gaussian processes, support vector machines, and neural networks which are commonly used non-linear models.

\subsubsection{Tree-based models}

As supervised learning models, tree-based models are nonparametric and can be used for both regression and classification. They can capture the non-linear relations between the predicting and target variables by splitting the data on certain feature values according to some conditions in a nested way. The idea behind each split is to create more homogeneous datasets within each split, where the target values within the split are more similar to each other than they are before the split. Thus, the conditions that are used for data splitting are determined by the potential homogeneity of target values.

In a regression task, the tree-based model will search through each distinct value of every feature, starting with the entire dataset, finding the feature and split value, and then partition the data into two subsets $\left(S_{1}\right.$ and $\left.S_{2}\right)$ such that the overall sum-of-square errors are minimized. Due to this mechanism, tree models can be used to determine the feature importance, since the most influential feature would be the first to split data on.

$$
S S E=\sum_{i \in S_{i}}\left(y_{i}-\bar{y}_{1}\right)^{2}+\sum_{i \in S_{2}}\left(y_{i}-\bar{y}_{2}\right)^{2}
$$

One of the biggest problems that decision trees have is overfitting. A single tree tends to overly fit the training set, causing poor generalization on unseen data. To address this problem, ensemble methods can be used, such as random forest ${ }^{[55]}$ and gradient boosting trees. ${ }^{[56]}$

Being nonlinear and non-parametric, tree-based regression models have many advantages. Firstly, tree-based models have excellent interpretability that decisions are made based on features in a certain order and their values. Secondly, treebased models can deal with both categorical and continuous input features naturally, requiring minimal data preparation. And finally, while tree-based models can capture complex non-linear relationships lying within the input-output pairs, they can automatically reflect the importance of input features. Therefore, tree-based models are widely used in predicting TE material properties from material descriptors. Carrete et al. 
adopted random forest regression to predict lattice thermal conductivity for half-Heusler compounds based on the chemical, compound, and specific thermal conductivity information. ${ }^{\left[{ }^{[7]}\right.}$ They further used the trained regression model to screen the thermodynamical stability. Gaultois et al. trained a random forest model to use the information contained in the periodic table to predict the Seebeck coefficient, thermal conductivity, electrical resistivity, and band gap. ${ }^{[58]}$ Also, the developed model successfully suggested an experimentally viable new compound from true chemical white space, which demonstrated the potential of using machine learning techniques to guide material discovery and design. Furmanchuk et al. utilized random forest to predict the Seebeck coefficient of crystalline materials, achieving fast property prediction for experimentally synthesized materials. ${ }^{[2]]}$ In addition to the linear model, Miller et al. used the random forest for predicting the carrier concentration range and dupability of diamond-like semiconductors. ${ }^{[52]}$ However, the random forest did not outperform the linear model for this task. Wu et al. used regression tree ensembles of LSBoost to predict the interfacial thermal resistance between two materials with curated physical, chemical, and material property descriptors. ${ }^{[59]}$ The ensemble model achieved a coefficient of determination $\left(R^{2}\right)$ of 0.919 with all descriptors and 0.907 with only property descriptors and thickness. Along with the implementation of the elastic net and quadratic polynomial Lasso regression under the framework of linear models, Iwasaki et al. also employed a decision tree regression model to predict the thermopower. ${ }^{[54]}$ The model was able to help explore the fundamental physics of the spindriven TE effect and improve materials with these effects. Wang et al. investigated the predictive power of the random forest regression model to estimate the figure of merit (ZT) of hot-extruded $\mathrm{Cu}_{\mathrm{x}} \mathrm{Bi}_{2} \mathrm{Te}_{2.85+\mathrm{y}} \mathrm{Se}_{0.15} \mathrm{TE}$ materials. ${ }^{[60]}$

Tree-based models are simple to understand, able to model the non-linearity in the data, and can deal with both continuous variables. Nevertheless, there are a few drawbacks to treebased models. Due to the way of growing the trees, tree-based models are sensitive to changes in training sets and thus are prone to overfitting, having relatively low bias but high variance. In addition, training an effective tree-based model requires a large amount of data since the tree-based models are not bounded by any assumption. Lastly, in a regression task, tree-based models return the average values of the data in the training set on leaves, making them relatively inadequate for predicting continuous values.

\subsubsection{Gaussian process}

Gaussian processes (GPs) are supervised learning models widely used in regression problems. Different from the techniques mentioned in the previous sections, Gaussian process regression models could innately quantify the prediction uncertainty. Gaussian processes assume that the distribution over the function values at an arbitrary and finite set of points is jointly Gaussian. More specifically, let $\mathbf{x}_{1}, \ldots, \mathbf{x}_{\mathbf{i}}$ be a set of values of the variable vector $\mathbf{x}$, and $f\left(\mathbf{x}_{1}\right), \ldots, f\left(\mathbf{x}_{\mathrm{i}}\right)$ the function values at these points. The distribution $p\left(f\left(\mathbf{x}_{1}\right), \ldots, f\left(\mathbf{x}_{\mathbf{i}}\right)\right)$ is a multivariate Gaussian with mean $\mu(\mathbf{x})$ and covariance $\sum(\mathbf{x})$. The covariance is calculated by $\Sigma_{\mathrm{ij}}=\kappa\left(\mathbf{x}_{\mathbf{i}}, \mathbf{x}_{\mathbf{j}}\right)$, where $\kappa(\cdot)$ is a positive definite kernel function. The kernel function measures the similarity between two data points which would reflect on the similarity between the function values of these points. In general, a GP defines a prior over functions that can be converted to a posterior over functions after incorporating the observed data. In a regression setting, let the regression function prior be a GP, denoted by:

$$
f(\mathbf{x}) \sim G P\left(m(\mathbf{x}), \kappa\left(\mathbf{x}, \mathbf{x}^{\prime}\right)\right)
$$

where $m(\mathbf{x})$ is the mean function and $\kappa\left(\mathbf{x}, \mathbf{x}^{\prime}\right)$ is the covariance function:

$$
\begin{gathered}
m(\mathbf{x})=E[f(\mathbf{x})] \\
\kappa\left(\mathbf{x}, \mathbf{x}^{\prime}\right)=E\left[f(\mathbf{x})-m(\mathbf{x})\left(f(\mathbf{x})-m\left(\mathbf{x}^{\prime}\right)\right)^{T}\right]
\end{gathered}
$$

With observed data points $X$, the process defines a joint Gaussian

$$
p(f \mid X)=\mathcal{N}(f \mid \mu, K)
$$

where $K_{i, j}=\kappa\left(\mathbf{x}_{\mathbf{i}}, \mathbf{x}_{\mathbf{j}}\right)$, and $\mu=\left(m\left(\mathbf{x}_{\mathbf{1}}\right), \ldots, m\left(\mathbf{x}_{\mathbf{n}}\right)\right)$. Like many other techniques, GPs could capture the non-linearity within the data. In addition, GPs use Equation (12) to make predictions on unseen data points. Since the prediction is a distribution, it can automatically quantify the uncertainty of the prediction, producing both mean and standard deviation. This property has a great advantage over the aforementioned models when the quantified prediction uncertainty is preferred. Another special property that GPs have is that based on the observed data, GPs produce a posterior distribution over the regression function, interpolating every training data point, making sure that the learned function is bound to satisfy the observations. This is different from other methods where the learned regression function aims to minimize the average error between the function values and the observed data points. These special properties of GPs have made them popular in many data-driven applications, including TE materials. Zhan et al. adopted a GP regression model to predict thermal boundary resistance (TBR) with a dataset that had experimentally measured TBR as the training set. ${ }^{[61]}$ The GP regression model had the best predictive power for TBR among other reported methods and helped identify the importance of film thickness in predicting TBR. Juneja et al. aimed to evaluate the log-scaled lattice thermal conductivity using a GP regression model based on simple descriptors, which included maximum phonon frequency, integrated Gruneisen parameter, average atomic mass, and volume of the unit cell. ${ }^{[62]}$ In this study, the GP regression model achieved much superior performance to the Slack model, having a small root mean square error of $\sim 0.21$. The success of the machine learning model in predicting lattice thermal conductivity indicated a reliable and accelerated search for a multitude of low and high thermal conductivity materials. In the previously mentioned work, $\mathrm{Wu}$ et al. also utilized the GP regression 
model to predict the interfacial thermal resistance, achieving the second-best performance among reported models.

GP regression models are powerful in learning underlying mappings between input and output data, quantifying uncertainty, and having fair interpretability. However, a good GP model often requires the proper choice of the covariance function, which needs a certain level of domain-dependent expertise. Furthermore, GP models are computationally expensive and suffer greatly from the curse of dimensionality. In predicting TE material properties, a large number of descriptors are often used, making GP models less a suitable choice for training an effective and efficient model.

\subsubsection{Support vector regression}

Similar to linear regression, support vector regression (SVR) models use a linear function to approximate the regression function as defined in Equation (3). However, instead of minimizing the squared error as in the linear regression framework, which can be greatly influenced by just one observation that falls far from the overall trend, SVR aims to the so-called $\epsilon$-insensitive loss function, shown in Equation (13). The motivation behind SVR is to minimize the effect of outliers on the regression equation. A threshold $\epsilon$ is used in taking into account the error contribution from the data points, where the data points with residuals within this threshold do not contribute to the overall loss.

$$
L_{\epsilon}(y, f(\mathbf{x}))=\left\{\begin{array}{cc}
0 & \text { if }|y-f(\mathbf{x})|<\epsilon \\
|y-f(\mathbf{x})|-\epsilon & \text { otherwise }
\end{array}\right.
$$

To estimate the model parameters, SVR also adds a penalty term. Thus, the complete objective function is denoted by Equation (14).

$$
J=C \sum_{i=1}^{n} L_{\epsilon}(y, f(\mathbf{x}))+\frac{1}{2}|| \beta \|^{2}
$$

One can show that for a data point, the prediction function is

$$
f(\mathbf{x})=\beta_{0}+\sum_{i} \alpha_{i} \mathbf{x}_{\mathbf{i}}^{\mathbf{T}} \mathbf{x}
$$

The dot product can be replaced by a kernel function $\kappa\left(\mathbf{x}_{\mathbf{i}}, \mathbf{x}\right)$, representing the dot product in higher dimensions, and thus capturing the non-linear relations within the data. SVR models are highly flexible and relatively robust against outliers. By the different choices of the kernel functions, SVR can perform both linear and non-linear regression tasks. In the application of predicting TE material properties, SVR presented good performance while maintaining efficiency. Along with generalized linear regression, Gaussian process, Zhan et al. ${ }^{[61]}$ also employed SVR to predict the thermal boundary resistance and achieved the best comparable results with the GP regression model. In the work of predicting interfacial thermal resistance ${ }^{[59]}$, Wu et al. used SVR with radial basis function (RBF) kernel-based on physical, chemical, and material properties. However, SVR produced inferior results to LSBoost and GP regression models. Wang et al. trained an SVR model on the experimental data to predict ZT and achieved good performance on the testing set.

SVR models have a few shortcomings. The choice of the kernel function when applying SVR to a non-linear problem is rather important. Similar to GP regression, there is not a universal optimal kernel function and the choice should be depending on the data. While the RBF kernel has been shown to be very effective, it would have worse performance than the linear kernel when the underlying problem is linear. In addition, as a more complex model, SVR has more hyperparameters that cannot be updated via learning from the data.

\subsubsection{Deep learning}

Deep learning methods, different from the classic machine learning techniques, are based on neural network theories, where operations can be represented by connected neurons. Based on the universal approximation theorem, ${ }^{[63]}$ artificial neural networks are adequate estimators for any functions. Instead of using a large number of neurons in a single layer to capture complex mapping within the data, multiple connecting layers of neurons could address the intractability problem while maintaining performance. Besides the ability of capturing sophisticated mappings, deep learning methods can learn multiple levels of representations of the original input data, which are obtained by non-linear modules transforming the representation one level at a time. ${ }^{[64]}$ The representation learning ability allows the rawest form of data and does not require much feature engineering and selection. In the application of TE materials, the feed-forward fully connected neural networks (FCNN) are mostly used. FCNNs are composed of multiple layers of neurons with an input layer, a few hidden layers, and an output layer, where neurons in one layer are connected with the neurons in successive layers. Between layers, a matrix multiplication operation takes place, assigning a weight to each input feature. Each neuron then outputs the summation of weighted feature values and sends them to a non-linear activation function. The output of the activation function is further sent to the next layer. The activation function is responsible for activating the neuron and introducing the non-linearity in the model. A schematic of an FCNN is shown in Fig. 5.

Due to the flexibility and predictive power of the FCNNs, and the natural form of material descriptors, these networks are used to predict TE material properties. Kolb et al. used FCNNs to develop PROPhet that could find complex and nonlinear mappings between sets of material or system properties. ${ }^{[65]}$ This framework could also be used to generate density functionals for system properties or potential energy surfaces. Miller et al. compared the performance of the FCNN with linear regression and random forest to predict the dopability range. ${ }^{[52]}$ In the work of predicting thermopower, ${ }^{[54]}$ an FCNN was used to map 4 descriptors to the output thermopower value with hyperbolic tangent and sigmoid functions as activation. Want et al. ${ }^{[60]}$ used a fully connected network to predict ZT.

Despite the advantages deep learning has over the classic machine learning models, it is often over-parameterized and thus requires a huge amount of data to be able to learn a reasonable mapping that generalizes well. This imposes an 


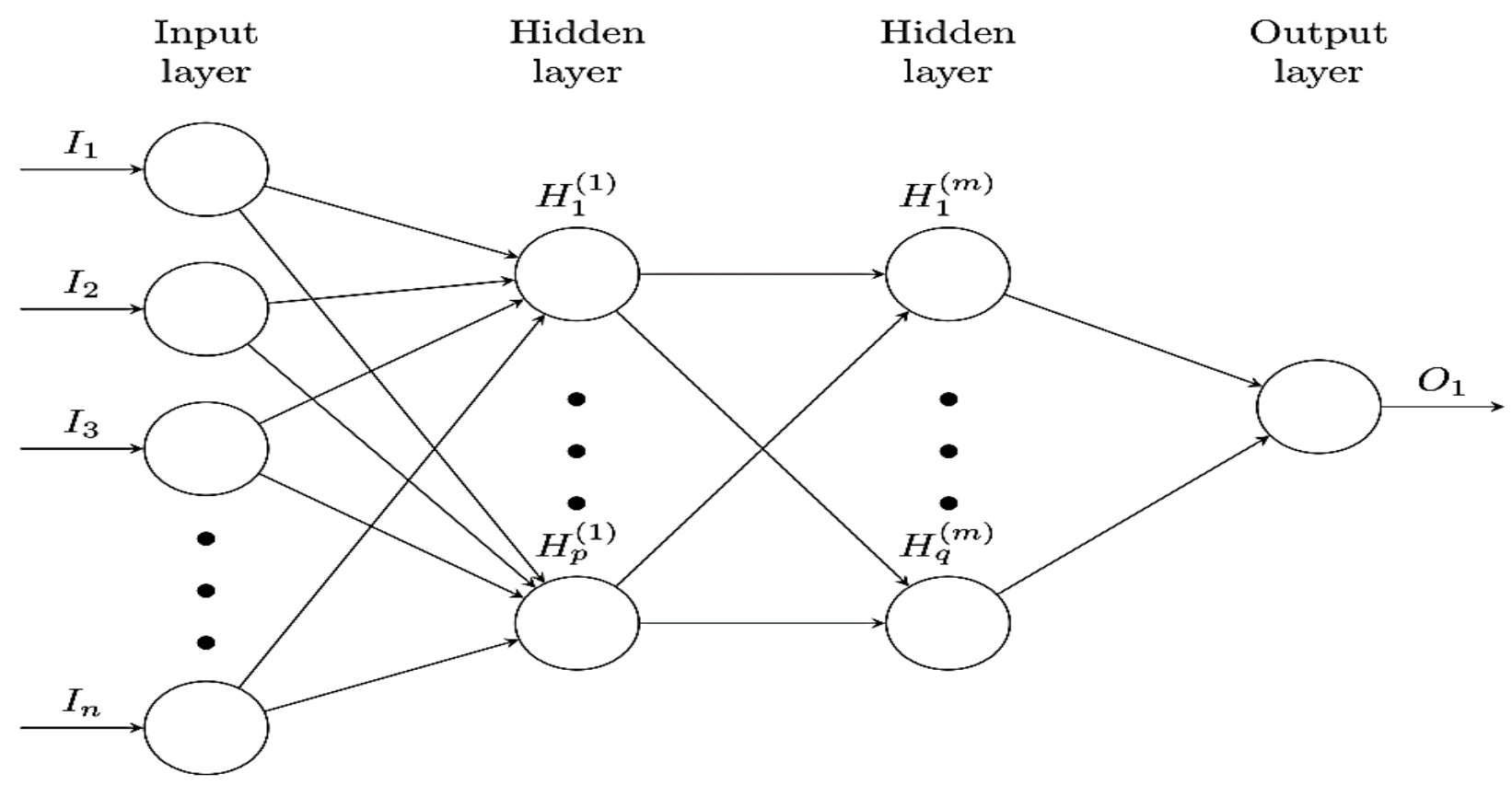

Fig. 5 A schematic of a general fully connected neural network. It consists of an input layer, one or more hidden layers, and an output layer. A weight is assigned to each connection between nodes in different layers. At each node, a summation operation and an optional activation function are placed.

important constraint when applying deep learning methods to predicting material properties because of the data scarcity and data sparsity. In addition, the interpretability of deep learning models is poor, due to the lack of pre-defined model form and complex hierarchy of layers and neuron activation.

\section{Evaluation Metrics}

Evaluating and comparing trained machine learning models for real applications are important, and it usually depends on the specific application requirement. For regression models, there are various ways of quantifying the goodness of fit, among which differences sometimes are significant enough to yield opposite conclusions. In this section, we describe the commonly used regression model evaluation metrics found in the existing TE material machine learning literature and state the proper situations of usage.

\section{$5.1 R^{2}$}

Coefficient of determination $\left(R^{2}\right)$ is one of the most commonly used metrics to evaluate the goodness of fit of a regression model. It was used for thermoelectric material machine learning model evaluation and comparison in many studies. ${ }^{[21,53,59,60,62]}$ It depicts the proportion of the variance in the true target values that can be explained by the regression model. The range of $R^{2}$ is from 0 to 1 but the values of $R^{2}$ can exceed 1 or be less than 0 when the model fits the data worse than a horizontal hyperplane, depending on the way of calculating. The most widely used formula to get $R^{2}$ is shown in Equation (16).

$$
R^{2}=1-\frac{S S_{r e s}}{S S_{t o t}}
$$

where $\mathrm{SS}_{\mathrm{res}}=(\mathrm{y}-\mathrm{f}(\mathbf{x}))^{2}$ and $\mathrm{SS}_{\text {tot }}=(\mathrm{y}-\overline{\mathrm{y}})^{2}$ being the mean of the true target values. $R^{2}$ has the interpretation of how much variation lying in the true target values can be explained by the regression model, resulting in an advantage of which the model performance in terms of $R^{2}$ is clear and straightforward regardless of the target range. However, this very advantage could cause problems when using $R^{2}$ to evaluate a regression model performance. Based on the definition, $R^{2}$ is dependent on both the sum of squared error and variance in the target values. Thus, it is possible to high $R^{2}$ with a poorly performing regression model while the target variance is large. Conversely, if the target variance is small, a well-performing model could still result in a small $R^{2}$, which might imply the opposite conclusion. Besides, while using $R^{2}$ is convenient to compare the performance of multiple regression models, it does not reflect the measure of predictive errors. The predictive errors are important in determining whether the regression model meets a certain accuracy requirement for specific tasks. Therefore, a better practice of using $R^{2}$ to evaluate models is to combine other regression metrics to avoid these pitfalls.

\subsection{RMSE}

The root mean squared error (RMSE), as a measure of the predictive errors, indicates the standard deviation of the residuals. It is defined in Equation (17)

$$
R M S E=\sqrt{\frac{\sum_{i=1}^{N}\left(y_{i}-f\left(\mathbf{x}_{\mathbf{i}}\right)\right)^{2}}{N}}
$$


where $N$ is the number of data points used for model evaluation. RMSE has the same scale as the original target values and bears the physical dimensions. A smaller RMSE implies better regression model performance. This metric was widely used in the current literature ${ }^{[21,53,54,59,61,62]}$ in comparing machine learning regression models. Different from the mean absolute error (MAE) which accounts for the averaged deviation of the model predictions from the true target values and will be talked about in the following section, RMSE is based on the squared point distance measure and arithmetic mean aggregation, which can be interpreted as the standard deviation of errors. Due to the square, same as in using mean squared error (MSE), the contribution of model prediction errors is not uniform, where larger errors have a relatively greater effect on the metric value. This can result in a misleading conclusion about model performance over the lower end of target values when the model makes a small magnitude of prediction error but having a high percentage error. Thus, RMSE or MSE can be used when there is an emphasis on using large errors to discriminate regression models.

\subsection{MAE}

The most natural way to measure the goodness of fit for a regression model is to calculate the prediction residuals. Nevertheless, the mutual cancellation of the positive and negative residuals can lead to seemingly good performance while the model makes inaccurate predictions. To address this issue, mean absolute error (MAE) can be adopted. It is defined in Equation (18).

$$
M A E=\frac{\sum_{i=1}^{N}\left|y_{i}-f\left(\mathbf{x}_{\mathbf{i}}\right)\right|}{N}
$$

MAE only considers how much the prediction deviates from its true value regardless of over- or under-prediction. It preserves the same units/scale of the observations and is easily interpretable. Unlike in the squared error-based metrics, the error contribution to MAE is proportional to its magnitude. Therefore, MAE is a more appropriate choice when small and large errors are considered equally important. Thanks to its outstanding interpretability, MAE was used in evaluating the performance of machine learning regression models in predicting TE material properties. ${ }^{[52]}$

\subsection{RAE}

RAE stands for relative absolute error which is defined in Equation (19).

$$
M A E=\frac{\sum_{i=1}^{N}\left|y_{i}-f\left(\mathbf{x}_{\mathbf{i}}\right)\right|}{\sum_{i=1}^{N}\left|y_{i}-\bar{y}\right|}
$$

Same as in MAE, RAE uses the absolute error as the point distance measure. However, instead of providing the value with the same units and physical meaning as MAE, RAE measures the ratio of the errors made by the regression model to the errors made by a trivial model. A good performing regression model is expected to have RAE close to 0 . The magnitude of RAE depends on both model prediction error and the variation that lies within the target values. Therefore, RAE is suitable for comparing models that serve the same task.

\subsection{RSE}

While RAE uses absolute error to be the point distance measure, relative squared error (RSE) uses squared error, putting more importance on the contribution made by large errors. It is defined in Equation (20).

$$
R S E=\frac{\sum_{i=1}^{N}\left(y_{i}-f\left(\mathbf{x}_{\mathbf{i}}\right)\right)^{2}}{\sum_{i=1}^{N}\left(y_{i}-\bar{y}\right)^{2}}
$$

RSE, similar to RAE, returns a ratio that represents the proportion of squared model prediction errors in the total sumof-squares of the target value. Due to the squared point distance measure, RAE is more sensitive to large errors, and therefore can be used in evaluating models when large errors are more important to avoid.

The choice of evaluation metrics for regression models is largely dependent on the problem and there is not a universally best metric. Thus, before adopting any evaluation metrics, one should have a clear picture of what aspect is more important in using the regression model.

\section{Conclusions}

This paper set out to review the recent progress in machine learning regression aided thermoelectric materials discovery. Learning from the relation between thermoelectricity and materials features, the machine learning technique can provide a powerful thermoelectric material discovery tool with respect to new chemical composition, nano-structural design, stoichiometry optimization, etc. This newly obtained information could be further utilized to enrich the thermoelectric database and improve the performance of the machine learning model training. The active learning approach is recommended for further exploration. The presented working structure can serve as a trusty reference for applying the AI-guided data-driven approaches in thermoelectric material discovery. Since most relevant studies were limited to using the machine learning technologies to explore and design materials with excellent thermoelectricity, other merits which are equally important, such as non-toxic and earth-abundant are suggested to be considered as the additional output of thermoelectric discovery tool. The presented work logic can also be utilized when developing the material discovery tool for properties other than thermoelectricity, such as photovoltaic, piezoelectricity, etc. More information on the fundamental correlation between machine learning algorithms and material science will help establish a greater degree of functionality on this matter. With the extraordinary contribution from the platform of opensources tools and publicly available databases, the machine learning-aided approach has a great potential to revolutionize materials discovery. 


\section{Acknowledgment}

$\mathrm{Na} \mathrm{Lu}$ is grateful for the financial supports from National Science Foundation (under Grants NSF CAREER 15604834; and NSF PFI-TT: 1919191). Guang Lin gratefully acknowledges the support from the National Science Foundation DMS-1555072.

\section{Supporting Information}

Not Applicable.

\section{Conflict of Interest}

There is no conflict of interest.

\section{References}

[1] Y. Feng, A. Elquist, Y. Zhang, K. Gao I. Ferguson' A. Tzempelikos, N. Lu, Compos. Part B, 2019, 156, 108-112, doi: 10.1016/j.compositesb.2018.08.070.

[2] W. Yang, Z. Chuanle, M. E. Aline, G. Amirhossein V. G. Saravade, N. Lu, I. Ferguson, Proc. SPIE, 2018, 10533, doi: 10.1117/12.2302467.

[3] Y. Feng, E. Witkoske, E. S. Bell, Y. Wang, A. Tzempelikos and I. T. Ferguson and N. Lu, ES Mater. Manuf., 2018, 1, 1320, doi: 10.30919/esmm5f116.

[4] T. Ye, X. Wang, X. li, A. Yan, S. Ramakrishna, and J. Xu, J. Mater. Chem. C, 2017, 5, 1255-1260, doi: 10.1039/C6TC04594D.

[5] Y. Feng, X. Jiang, E. Ghafari, B. Kucukgok, C. Zhang, I. Ferguson \& N. Lu, Adv. Compos. Hybrid Mater, 2018, 1, 114 126, doi: 10.1007/s42114-017-0011-4.

[6] E. Witkoske, Z. Tong, Y. Feng, X. Ruan, M. Lundstromand N. Lu, J. Appl. Phys., 2020, 127, 115108, doi: $10.1063 / 1.5142485$.

[7] Q. Hao, N. Yang, N. Lu, X. Ruan, Front. Energy. Res., 2019, 7, 109, doi: 10.3389/fenrg.2019.00109.

[8] E. Witkoske, D. Guzman, Y. Feng, A. Strachan, M. Lundstromand N. Lu, J. Appl. Phys., 2019, 125, 082531, doi: 10.1063/1.5058275.

[9] M. Schmidt, H. Lipson, Science, 2009, 324, 81-85, doi: 10.1126/science.1165893.

[10] T. Moot, O. Isayev, R. W. Call, S. M. McCullough, M. Zemaitis, R. Lopez, J. F. Cahoon, A. Tropsh, Mater. Discov, 2016, 6, 9-16, doi: 10.1016/j.md.2017.04.001.

[11] G. Lin, N. Lu, ES Mater. Manuf., 2020, 9, 1-2, doi: 10.30919/esmm5f915.

[12] C.-T. Chen, G. X. Gu, MRS Commun., 2019, 9, 556-566, doi: $10.1557 / \mathrm{mrc} .2019 .32$.

[13] G. R. Schleder, A. C. M. Padilha, C. M. Acosta, M. Costa, A. Fazzio, J. Phys. Mater, 2019, 2, 032001, doi: 10.1088/2515-7639/ab084b.

[14] T. Wang, C. Zhang, H. Snoussi, G. Zhang, Adv. Funct. Mater., 2020, 30, 1906041, doi: 10.1002/adfm.201906041.

[15] B. Kucukgok, Q. He, A. Carlson, A. G. Melton, I. Ferguson \& N. Lu, MRS Proc., 2013, 1490, 161-166, doi: 10.1557/opl.2013.26.
[16] E. N. Hurwitz, B. Kucukgok, A. G. Melton, Z. Liu, I. Ferguson \& N. Lu, MRS Proc., 2012, 1396, mrsf11-1396o1308-1310, doi: 101557/opl.2012.89.

[17] M. W. Gaultois, T. D. Sparks, C. K. H. Borg, R. Seshadri, Chem. Mater., 2013, 25, 2911-2920, doi: 10.1021/cm400893e. [18] A. o. Furmanchuk, J. E. Saal, J. W. Doak, G. B. Olson, A. Choudhary A. Agrawal, J. comput. chem., 2018, 39, 191-202, doi: $10.1002 /$ jcc. 25067 .

[19] Z. L. Wang, Y. Yokoyama, T. Onda, Y. Adachi, Z.-C. Chen, Adv. Electron. Mater., 2019, 5, 1900079, doi: 10.1002/aelm.201900079.

[20] B. Wang, B. Kucukgok, Q. He, A. G. Melton, J. Leach, K. Udwary, K. Evans, N. Lu \& Ian T. Ferguson MRS Online Proc. Libr., 2013, 1558, 903, doi: 10.1557/opl.2013.1136.

[21] J. Li, J. Sui, Y. Pei, X. Meng, D. Berardan, N. Dragoe, W. Caia and L. Zhao, J. Mater. Chem. A, 2014, 2, 4903-4906, doi: 10.1039/C3TA14532H.

[22] D. Zhang, J. Yang, Q. Jiang, L. Fu Y. Xiao, Y. Luo, Z. Zhou, Mater. Des., 2016, 98, 150-154, doi: 10.1016/j.matdes.2016.03.001.

[23] B. Zhou, S. Li, W. Li, J. Li, X. Zhang, S. Lin, Z. Chen, and Y. Pei, ACS Appl. Mater. Interfaces, 2017, 9, 34033-34041, doi: 10.1021/acsami.7b08770.

[24] Z. Hou, Y. Takagiwa, Y. Shinohara, Y. Xu, K. Tsuda, ACS Appl. Mater. Interfaces, 2019, 11, 11545-11554, doi: 10.1021/acsami.9b02381.

[25] M. Sasaki, S. Ju, Y. Xu, J. Shiomi, M. Goto, ACS Comb. Sci., 2020, 22, 782-790, doi: 10.1021/acscombsci.0c00112.

[26] W. Li, J. Carrete, N. A. Katcho, N. Mingo, Comput. Phys. Commun., 2014, 185, 1747-1758, doi: 10.1016/j.cpc.2014.02.015.

[27] G. K. H. Madsen, D. J. Singh, Comput Phys Commun., 2006, 175, 67-71, doi: 10.1016/j.cpc.2006.03.007.

[28] S. Wang, Z. Wang, W. Setyawan, N. Mingo, S. Curtarolo, Phys. Rev. X, 2011, 1, 021012, doi: 10.1103/PhysRevX.1.021012.

[29] Q. Hao, D. Xu, N. Lu, H. Zhao, Phys. Rev. B, 2016, 93, 205206, doi: 10.1103/PhysRevB.93.205206.

[30] S. Curtarolo, G. L. W. Hart, M. B. Nardelli, N. Mingo, S. Sanvito and O. Levy Nat. Mater., 2013, 12, 191-201, doi: 10.1038/nmat3568.

[31] J. Greeley, T. F. Jaramillo, J. Bonde, I. Chorkendorff, r. No, Jens K., Nat. Mat., 2006, 5, 909-913, doi: $10.1038 /$ nmat 1752 .

[32] S. Bhattacharya, R. Chmielowski, G. Dennler, G. K. H. Madsen, J. Mater. Chem. A, 2016, 4, 11086-11093, doi: 10.1039/C6TA04104C.

[33] Z. Liu, B. Fu, X. Yi, G. Yuan, J. Wang, Jinmin Li, Lun, and Ian Ferguson $R S C A d v$, 2016, 6, 5111-5115, doi: 10.1039/C5RA24642C.

[34] F. Ricci, W. Chen, U. Aydemir, G. J. Snyder, Sci. Data, 2017, 4, 170085, doi: 10.1038/sdata.2017.85.

[35] W. Chen, J.-H. Pöhls, G. Hautier, D. Broberg, S. Bajaj, U. Aydemir, Z. M. Gibbs, H. Zhu, M. Asta, G. J. Snyder, B. 
Meredig, M. White, K. Perssonae and A. Jain, J. Mater. Chem. C, 2016, 4, 4414-4426, doi: 10.1039/C5TC04339E.

[36] K. Choudhary, K. F. Garrity, F. Tavazza, J. PhysCondens. Mat., 2020, 32, 475501, doi: 10.1088/1361648x/aba06b.

[37] L. M. Ghiringhelli, J. Vybiral, S. V. Levchenko, C. Draxl, M. Scheffler, Phys. Rev. Lett., 2015, 114, 105503, doi: 10.1103/PhysRevLett. 114.105503.

[38] C. Chen, W. Ye, Y. Zuo, C. Zheng, and S. P. Ong, Chem. Mater. $\quad 2019, \quad$ 9, 3564-3572, doi: 10.1021/acs.chemmater.9b01294.

[39] F. Faber, A. Lindmaa, O. A. von Lilienfeld, R. Armiento, Int. J. Quantum Chem., 2015, 115, 1094-1101, doi: 10.1002/qua.24917.

[40] L. Himanen, M. O. J. Jäger, E. V. Morooka, F. Federici Canova, Y. S. Ranawata, D. Z. Gao, P. Rinke, A. S. Foster, Comput. Phys. Commun., 2020, 247, 106949, doi: 10.1016/j.cpc.2019.106949.

[41] J. He, T. M. Tritt, Science, 2017, 357, doi: 10.1126/science.aak9997.

[42] T. Wang, C. Zhang, H. Snoussi, G. Zhang, Adv. Funct. Mater., 2020, 30, 1906041, doi: 10.1002/adfm.201906041.

[43] L. Bassman, P. Rajak, R. K. Kalia, A. Nakano, F. Sha, J. Sun, D. J. Singh, M. Aykol, P. Huck, K. Persson and P. Vashishta, npj Comput. Mater., 2018, 4, 74, doi: 10.1038/s41524-018-0129-0.

[44] A. O. Oliynyk, E. Antono, T. D. Sparks, L. Ghadbeigi, M. W. Gaultois, B. Meredig, and A. Mar, Chem. Mater., 2016, 28, 7324-7331, doi: 10.1021/acs.chemmater.6b02724.

[45] K. Choudhary, B. DeCost, F. Tavazza, Phys. Rev. Materials, 2018, 2, 083801, doi: 10.1103/PhysRevMaterials.2.083801.

[46] S. Wang, Z. Wang, W. Setyawan, N. Mingo, S. Curtarolo, Phys. Rev. X, 2011, 1, 021012, doi: 10.1103/PhysRevX.1.021012.

[47]"A tutorial on Principal Components Analysis, "Computer Science Technical Report, (L. I. Smith, 2012).

[48] A. van Roekeghem, J. u. Carrete, C. Oses, S. Curtarolo, N. Mingo, Phys. Rev. X, 2016, 6, 041061, doi: 10.1103/PhysRevX.6.041061.

[49] N. Wagner, J. M. Rondinelli, Front. Mater., 2016, 3, 28, doi: $10.3389 /$ fmats. 2016.00028 .

[50] E. Kim, K. Huang, S. Jegelka, E. Olivetti, npj Comput. Mater. , 2017, 3, 1-9, doi: 10.1038/s41524-017-0055-6.

[51] M. De Jong, W. Chen, R. Notestine, K. Persson G. Ceder, A. Jain, M. Asta and A. Gamst, Sci. Rep., 2016, 6, 34256, doi: $10.1038 /$ srep34256.

[52] S. A. Miller, M. Dylla, S. Anand, K. Gordiz, G. J. Snyder and E. S. Toberer npj Comput. Mater., 2018, 4, 1-8, doi: 10.1038/s41524-018-0123-6.

[53] Z. Li, Q. Xu, Q. Sun, Z. Hou, W.-J. Yin, Adv. Funct. Mater., 2019, 29, 1807280, doi: 10.1002/adfm.201807280.

[54] Y. Iwasaki, I. Takeuchi, V. Stanev, A. G. Kusne, Sci. Rep., 2019, 9, 1-7, doi: 10.1038/s41598-019-39278-z.
[55] L. Breiman, Mach. Learn., 2001, 45, 5-32, doi: 10.1023/A:1010933404324.

[56] J. H. Friedman, Ann. stat, 2001, 1189-1232, doi: 10.1214/aos/1013203451.

[57] J. u. Carrete, s, W. Li, N. Mingo, S. Wang, S. Curtarolo, Phys. Rev. X, 2014, 4, 011019, doi: 10.1103/PhysRevX.4.011019.

[58] M. W. Gaultois, A. O. Oliynyk, A. Mar, T. D. Sparks, G. J. Mulholland, and B. Meredig, Apl Mater., 2016, 4, 053213, doi: 10.1038/s41524-019-0193-0.

[59] Y.-J. Wu, L. Fang, Y. Xu, npj Comput. Mater, 2019, 5, 56, doi: 10.1063/1.4952607.

[60] Z.-L. Wang, Y. Adachi, Z.-C. Chen, Adv. Theory Simul., 2020, 3, 1900197, doi: 10.1002/adts.201900197.

[61] T. Zhan, L. Fang, Y. Xu, Sci. Rep., 2017, 7, 1-9, doi: 10.1038/s41598-017-07150-7.

[62] R. Juneja, G. Yumnam, S. Satsangi, A. K. Singh, Chem. Mater. $\quad 2019, \quad$ 31, 5145-5151, doi: 10.1021/acs.chemmater.9b01046.

[63] K. Hornik, M. Stinchcombe, and H. White, Neural Networks, 1989, 2, 359-366, doi: 10.1016/08936080(89)90020-8.

[64] Y. LeCun, Y. Bengio, G. Hinton, Nature, 2015, 521, 436444, doi: 10.1038/nature14539.

[65] B. Kolb, L. C. Lentz, A. M. Kolpak, Sci. Rep., 2017, 7, 19, doi: 10.1038/s41598-017-01251-z.

\section{Author information}

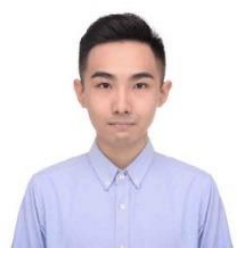

Guangshuai Han is a Ph.D. student in Civil Engineering at Purdue University. His current research interest includes flexible piezoelectric device, infrastructure sensing, and data-driven materials discovery.

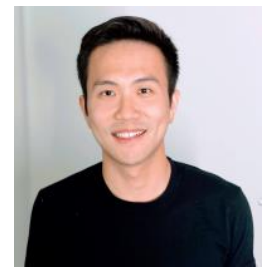

Yixuan Sun is a Ph.D. student in the school of mechanical engineering at Purdue University. His work focuses on combining data-driven methods, especially deep learning techniques with physical principles to solve engineering problems in material discovery and design, power and traffic networks, and fluid mechanics.

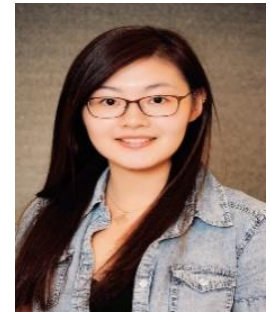

Yining Feng is a Postdoc Research Associate at the Lyles School of Civil Engineering, Purdue University. Her current research area focuses on flexible sensors for biological and environmental related applications. Yining received a Ph.D. in Civill Engineering from Purdue University. She has published one book chapter, 14 journal papers and three conference proceedings. 


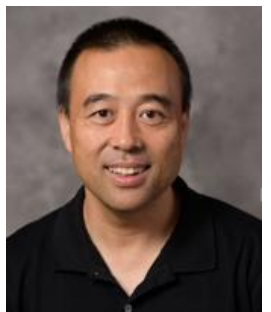

Guang Lin received a B.S. degree in mechanics from Zhejiang University in 1997, a M.S. degree in mechanics and engineering science from Peking University, and a Ph.D. degree from the Division of Applied Mathematics, Brown University in 2007. Currently he is an associate professor at both School of Mechanical Engineering and Department of Mathematics at Purdue University. Guang Lin received NSF faculty early career development award. His research interests include machine learning, uncertainty quantification, optimization, Bayesian algorithms and big data analysis.

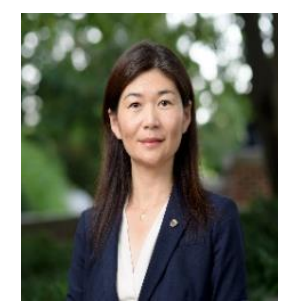

$\mathrm{Na} \mathbf{L u}$ is ACPA Professor of the Lyles School of Civil Engineering and the founding director of Center for Intelligent Infrastructure (CII) at Purdue University. Her research program integrates civil engineering, electrical engineering, and materials science disciplines with three main topic areas: 1) thermoelectric materials for waste heat recovery, 2) piezoelectric materials for energy harvesting and sensing and 3) multi-functional infrastructure materials. She has authored over 100 peer-reviewed publications: 2 book, 6 book chapters, 1 patent and 6 provisional patents. She has won several scientific awards, including the 2014 NSF CAREER Award, 2018 Purdue Zimmerman Innovation Award, 2020 Vebleo Scientist Award and 2021 ASCE GameChanger etc.

Publisher's Note Engineered Science Publisher remains neutral with regard to jurisdictional claims in published maps and institutional affiliations. 